\title{
Análise da performance de um modelo de coluna simples utilizando diferentes parametrizações para a taxa dissipação viscosa de energia cinética turbulenta
}

\author{
Analysis of the single column model performance using different \\ parameterizations for the viscous dissipation rate of turbulence kinetic energy
}

\author{
Janete Gonçalves Lira, Eduardo Stüker, Jean Jonathan Schuster, Cristiano Henrique Schuster, \\ Daniel Michelon dos Santos, Luiz Eduardo Medeiros, Felipe Denardin Costa \\ Programa de Pós Graduação em Engenharia, Universidade Federal do Pampa, Alegrete, RS, Brasil. \\ janete.glira@gmail.com; eduardostuker@gmail.com; jeanjschuster@gmail.com, cris.h.schuster@gmail.com; \\ danielmichelon@gmail.com; luizmedeiros@unipampa.edu.br; fdenardin@unipampa.edu.br;
}

\begin{abstract}
Resumo
A descrição apropriada do escoamento atmosférico da camada limite estável é uma das tarefas mais complexas a serem executadas por modelos numéricos de previsão de tempo e clima. A maioria das parametrizações utilizadas nos modelos atmosféricos são baseadas na teoria estatística da turbulência em sua concepção. Entretanto, esta teoria é válida somente para um escoamento no qual a turbulência é homogênea, isotrópica e estacionária, condições que comumente não são satisfeitas durante a noite. Basicamente, o escoamento noturno pode ser classificado em dois regimes: um regime muito estável, onde a turbulência é quase que totalmente suprimida; e um regime pouco estável, caracterizado pela intensa mistura turbulenta. A transição entre estes regimes é conhecida como acoplamento atmosférico e pode se suceder inúmeras vezes durante uma mesma noite. Neste trabalho, é implementado um modelo de coluna simples com fechamento de turbulência 1.5, em três configurações e com três formulações de turbulência diferentes. Em geral, os resultados da validação do modelo mostram que todas as configurações são capazes de reproduzir as características médias do escoamento em condições de fraca estabilidade.
\end{abstract}

Palavras-chave: Camada Limite Estável, Turbulência, dissipação viscosa, Modelo de Coluna Simples.

\begin{abstract}
The proper description of the atmospheric flow in the stable atmospheric boundary layer is one of the most complex tasks to be performed by numerical models of weather and climate prediction. Most of the parameterizations used in these models are based on the statistical theory of turbulence in their conception. However, this theory is valid only for a flow in which turbulence is homogeneous, isotropic and stationary, a conditions that are not commonly found overnight. Basically, the nighttime flow can be classified in two major regimes: very stable, where turbulence is almost entirely suppressed; and weakly stable regime, with intense turbulent mixing. The transition between these regimes is known as atmospheric coupling, and it can happens a lot of times along the same night. In this work, we implemented a single column model with turbulence closure 1.5, in three different configurations and three different turbulence formulations. In general, the model results show that, all the configurations are able to reproduce the average characteristics of the flow in the weakly stable conditions.
\end{abstract}

Keywords: Stable Boundary Layer, Turbulence, vicous dissipation Single Column Model 


\section{Introdução}

O estudo das variáveis que controlam o escoamento atmosférico na região inferior da atmosfera é um tema muito importante em várias áreas do conhecimento, pois nesta região é onde ocorre a maioria das atividade humanas. Assim, o entendimento e representação adequada do escoamento atmosférico colabora para o sucesso de áreas como meteorologia, que está mais relacionada com o tema, passando por agricultura, comércio, até logística; uma vez que a representação do escoamento, nesta região, permite avanços na previsão do tempo que implicarão diretamente no planejamento e no sucesso de outras áreas.

A maioria dos modelos numéricos utilizados na descrição do escoamento atmosférico faz uso da teoria estatística da turbulência na sua concepção, já que seu uso permite uma série de simplificações nas equações de Navier-Stokes. Entretanto, essa teoria é válida somente para um escomento no qual a turbulência é homogênea, isotrópica e estacionária. Na CLP, no período diurno, as condições necessárias para se utilizar a teoria estatítica da turbulência são satisfeitas, porém, durante a noite, não é possível fazer a mesma afirmação.

No período noturno, a superfície do solo se resfria através da emissão de radiação de onda longa, resfriando, consecutivamente, a camada de ar adjacente a ela e, assim, dando início a um processo de estratificação térmica responsável pela redução das escalas temporais e espacias com intensidade turbulenta em várias ordens de grandeza. Nessa situação, as condições de isotropia e homogeneidade são respeitadas e, assim, a grande maioria dos modelos de turbulência falha ao descrever um escoamento nestas condições. E, consecutivamente, os modelos numéricos que utilizam essa teoria sucumbem frente a tal desafio. Nestas condições, a geração de turbulência é puramente mecância e, na maioria das parametrizações para a taxa de dissipação viscosa, esse fato não é considerado.

Desta forma, a meta deste trabalho é analisar a influência das parametrizações para a taxa de dissipação viscosa em um modelo de coluna simples, com ordem de fechamento 1.5 , e comparar a performance do modelo com os resultados de uma simulação utilizando a técnica de simulação de grades turbilhões (LES, do inglês Large Eddy Simulation), para uma camada limite estável (CLE) idealizada realizada por Kosovic e Curry (2000).

\section{Metodologia}

\subsection{Modelo}

\subsubsection{Equações prognósticas}

O fechamento de primeira ordem consiste em resolver as equações prognósticas para as componentes horizontais da velocidade do vento e para a temperatura, com os fluxos turbulentos sendo parametrizados utilizando teoria K. Neste trabalho, são implementados modelos com ordem de fechamento 1.5, esta ordem de fechamento implica resolver prognosticamente a ECT, que será usada na determinação dos coeficientes de difusão. Desta forma, o conjunto de equações que formam o modelo podem ser escritas como:

$$
\begin{gathered}
\frac{\partial \bar{u}}{\partial t}=f\left(\bar{v}-\overline{v_{G}}\right)+\frac{\partial}{\partial z}\left(K_{m} \frac{\partial \bar{u}}{\partial z}\right), \\
\frac{\partial \bar{v}}{\partial t}=f\left(\overline{u_{G}}-\bar{u}\right)+\frac{\partial}{\partial z}\left(K_{m} \frac{\partial \bar{v}}{\partial z}\right), \\
\frac{\partial \bar{\theta}}{\partial t}=\frac{\partial}{\partial z}\left(K_{h} \frac{\partial \bar{\theta}}{\partial z}\right) .
\end{gathered}
$$

Nas equações acima: $-\overline{u^{\prime} w^{\prime}}=K_{m} \frac{\partial \bar{u}}{\partial z},-\overline{v^{\prime} w^{\prime}}=K_{m} \frac{\partial \bar{v}}{\partial z}$ e $-\overline{w^{\prime} \theta^{\prime}}=\frac{\partial}{\partial z}\left(K_{h} \frac{\partial \bar{\theta}}{\partial z}\right)$, são os gradientes verticais das componentes do fluxo de momentum e energia na forma de calor, respectivamente. $K_{m}=\sqrt{\alpha \bar{e}} l_{m}$ e $K_{h}=K_{m} / P r_{t}$ são os coeficientes de difusão de momentum e de difusão de energia na forma de calor, nesta ordem. Inicialmente, o número de Prandlt turbulento é tomado como sendo $P r_{t}=0,85$, a critério de comparação dos resultados das simulações com os dados do experimento GABLES I (Cuxart et al., 2006). A constante $\alpha$ comumente é usada como um parâmetro da camada superfícial, todavia, Rodrigo e Anderson (2013) e Acevedo et al. (2014) mostram que entre ECT e a $\tau / \rho$ obedece a uma função fortemente dependente da estabilidade para diferentes alturas, inclusive, em níveis superiores ao topo da camada superfícial.

As funções que descrevem o comportamento de $\alpha$ propostas por Rodrigo e Anderson (2013) e por Acevedo et al. (2014) são bastante similares, todavia, no primeiro trabalho, foram utilizados dados provenientes de um sítio apenas, localizado na Antártica, enquanto que, no segundo trabalho, foram utilizados dados de 6 sítios com características superficiais completamente distintas: plantação de milho (Cruz Alta - RS, Brasil (Teichrieb et al., 2013)) ; neve (Experimento FLOSS II realizado em Walden - CO, EUA (Mahrt e Vickers, 2005)) ; pastagem (Experimento LBA, km77 Santarém - PA, Brasil (Sakai et al., 2004)) ; floresta tropical (Experimento LBA, Manaus, AM, Brasil (Araújo et al., 2002) e Uatumã - AM, Brasil); e floresta de araucárias (São João do Triunfo - 
PR, Brasil (Oliveira et al., 2013)). Além disso, as análises feitas por Acevedo et al. (2014) desconsideram a contribuição de fenômenos não turbulentos, com escalas de tempo maiores que a turbulência e menores que os fenômenos de mesoescala, referidos comumente como submeso (Mahrt, 2009; Belušić e Güttler, 2010). Como esta função é oriunda de uma análise mais completa e que não possui discrepâncias com as funções obtidas em outros trabalhos, está utilizada aqui, e é escrita como:

$$
\alpha=\left(4(1+2,5 z / \Lambda)^{1 / 3}\right)^{-1}
$$

Como anteriormente discutido, nos modelos de ordem 1.5, é necessário resolver uma equação para ECT, pois esta grandeza é utilizada na estimativa dos coeficientes de difusão. Para uma atmosfera seca, horizontalmente homogênea, não subsidente (ou seja $\bar{w}=0$ ), e sem divergência de fluxo radiativo, a equação prognóstica para a energia cinética turbulenta pode ser escrita como (Stull, 1988):

$$
\begin{array}{r}
\frac{\partial \bar{e}}{\partial t}=-\overline{u^{\prime} w^{\prime}} \frac{\partial u}{\partial z}-\overline{v^{\prime} w^{\prime}} \frac{\partial v}{\partial z}+\frac{g}{\Theta} \overline{w^{\prime} \theta^{\prime}}- \\
\frac{\partial}{\partial z}\left[\left(\overline{w^{\prime} e^{\prime}}\right)-\frac{\overline{p^{\prime} w^{\prime}}}{\rho_{0}}\right]-\varepsilon .
\end{array}
$$

Sendo o termo do lado esquerdo o balanço local da ECT, os dois primeiros termos do lado direito são os termos de produção por cisalhamento, o terceito termo é o termo de produção/destruição de turbulência por empuxo, os termos entre colchetes são os termos de transporte turbulento e transporte por flutuações de pressão e, finalmente, o último termo é a taxa de dissipação viscosa de ECT.

Os termos de cisalhamento e empuxo da equação (5) são parametrizados utilizando teoria K, a mesma aproximação é utilizada pelos termos de transporte. Embora o termo de transporte por flutuações de pressão não possa ser parametrizado por uma relação fluxo gradiente, muitos trabalhos costumam negligenciar sua influência, enquanto outros mostram que sua magnitude é considerável (Puhales et al., 2010). Neste trabalho, estes termos são parametrizados de acordo com o proposto por Duynkerke (1988):

$$
-\left[\left(\overline{w^{\prime} e^{\prime}}\right)-\frac{\overline{p^{\prime} w^{\prime}}}{\rho_{0}}\right]=K_{e} \frac{\partial \bar{e}}{\partial z},
$$

onde $K_{e}=K_{m} / \sigma_{e}$ é o coeficiente de difusão da ECT. $\mathrm{O}$ valor de $\sigma_{e}$ é escolhido como igual a uma unidade por dois motivos principais, o primeiro é que não existe consenso sobre o valor desta contante, já que é praticamente impossível de medir seu valor (Costa et al., 2011; Yuji et al., 2013). O segundo motivo é que grande parte das parametrizações utilizadas em modelos operacionais utiliza esta constante como sendo uma unidade.
Além disso, desta forma, a sensibilidade dos resultados a mais um parâmetro arbitrário é desprezada. Finalmente, tem-se o termo mais complexo para ser estimado, a dissipação viscosa de energia cinética turbulenta $\varepsilon$.

\subsubsection{Dissipação viscosa de ECT}

Tradicionalmente, a dissipação viscosa é parametrizada utilizando a equação de Kolmogorov que é obtida a partir da teoria estatítica da turbulência (TET) (Kolmogorov, 1941). O principal problema, neste caso, é que a TET é válida somente quando a turbulência no escoamento é homogênea, isotrópica e estacionária, condições que comumente não são observadas em escoamentos estratificados. Muitos modelos atmosféricos utilizam esta equação, todavia, a anisotropia e não homogeneidade do escoamento é representada por uma constante $c_{\mathcal{E}}$. Entretanto, em muitos casos, esta constante se torna um parâmetro de ajuste arbitrário, servindo para reduzir ou aumentar a mistura turbulenta do escoamento, podendo variar até uma ordem de magnitude, em um intervalo de valores que entre 0,07 e 0,7 (Cuxart et al., 2006). Para manter a coerência nas constantes e com o objetivo de indicar a influência do cisalhamento na taxa de dissipação viscosa, a constante que multiplica a taxa de dissipação é tomada como $c_{\text {varepsilon }}=\alpha^{3 / 2}$. Assim, a equação para a dissipação viscosa é escrita como:

$$
\varepsilon=\frac{(\alpha \bar{e})^{3 / 2}}{l_{d}},
$$

sendo $l_{d}$ o comprimento de mistura para a dissipação. Por simplicidade, em todos os casos aqui mostrados, quando utilizado, o comprimento de mistura para a dissipação é tomado como $l_{d}=l_{m}$. Para não ficar restrito a uma parametrização comumente utilizada, neste trabalho, serão utlizadas mais duas parametrizações para a $\varepsilon$, que serão descritas a seguir.

Os modelos que utilizam uma equação prognóstica para a taxa de dissipação de ECT que, quando utilizadas em um modelo de fechamento de ordem 1.5, o coeficiente de difusão de momentum é escrito como: $K_{m}=(\alpha \bar{e})^{2} / \varepsilon$. A equação para a dissipação que é classicamente utilizada é escrita como (Duynkerke, 1988; Weng e Taylor, 2003, 2006):

$$
\begin{array}{r}
\frac{\partial \varepsilon}{\partial t}=\frac{\varepsilon}{\bar{e}} c_{\varepsilon_{1}}\left[-\overline{u^{\prime} w^{\prime}} \frac{\partial u}{\partial z}-\overline{v^{\prime} w^{\prime}} \frac{\partial v}{\partial z}+\frac{g}{\Theta} \overline{w^{\prime} \theta^{\prime}}\right]+ \\
\frac{\partial}{\partial z}\left(c_{\varepsilon} \frac{\partial \varepsilon}{\partial z}\right)-c_{\varepsilon_{2}} \frac{\varepsilon^{2}}{\bar{e}} .
\end{array}
$$

Na equação acima, o termo do lado esquerdo da igualdade é o balanço local de dissipação. Já do lado direito, o primeiro termo é a produção de dissipação, o segundo termo é o transporte turbulento de dissipação e o último termo é a dissipação de dissipação viscosa. Apesar de, 
supostamente, descrever o escoamento de forma mais realista, quando uma equação prognóstica para $\varepsilon$ é utilizada, os modelos costumam simular uma CLE mais profunda do que a realidade (Weng e Taylor, 2003). A partir disso, modificações são realizadas para minimizar este fato, uma delas é a mudança na parametrização dos termos de produção de dissipação (Detering e Etling, 1985; Xu e Taylor, 1997), sendo a equação (8) reescrita como:

$$
\frac{\partial \varepsilon}{\partial t}=c_{\varepsilon_{1}} \frac{(\alpha \bar{e})^{3}}{l_{d}^{2}}+\frac{\partial}{\partial z}\left(c_{\varepsilon} \frac{\partial \varepsilon}{\partial z}\right)-c_{\varepsilon_{2}} \frac{\varepsilon^{2}}{\bar{e}} .
$$

Por ser estabelecido que a utilização da equação (8) resulta em dados não satisfatórios, aqui, somente a equação (9) é utilizada. Os valores das constantes são os mesmos propostos por (Weng e Taylor, 2003), são eles: $c_{\varepsilon_{1}}=1,44, c_{\mathcal{E}_{2}}=1,92$ e $c_{\varepsilon}=\alpha\left(c_{\mathcal{E}_{2}}-c_{\varepsilon_{1}}\right) / \kappa^{2}$. Além disso, outra modificação foi utilizada, neste trabalho, para evitar erros numéricos em condições muitos estáveis, quando ambas, ECT e a sua dissipação tendem a zero. Grande parte das formulações são utilizadas e testadas em condições de estabilidade próximas do limite neutro, que permite que a parametrização de $K_{m}$ característica de modelos $E-\varepsilon$ seja utilizada. Assim, $K_{m}$ é determinado da mesma forma que em um modelo $E-l$.

Um aspecto importante sobre as equações prognósticas para a taxa de dissipação é que estas não são muito precisas quando utilizadas em modelos numéricos pelo fato descrito acima, além disso, o significado físico de seus termos é de difícil entendimento (Hunt et al., 1987). Em condições muito estáveis, o escoamento turbulento é puramente mecânico e governado pelo cisalhamento, nesse contexto, a influência da distância da superfície e do cisalhamento do vento sobre os turbilhões é um ponto crucial na descrição do comportamento da turbulência. Neste sentido, Hunt et al. (1987) propuseram uma parametrização para $l_{d}$, baseada no experimento realizado por Hunt (1984) que descreve a influência da superfície e do cisalhamento sobre os turbilhões. Assim a equação de Kolmogorov pode ser reescrita como:

$$
\varepsilon=A_{B} \frac{\bar{e}^{3 / 2}}{l_{d}}+A_{s} \bar{e} \frac{\partial \bar{U}}{\partial z} .
$$

Na equação acima $A_{B}$ e $A_{S}$ são constantes experimentais com valores iguais a 0,27 e 0,46 e $\bar{U}$ é a magnitude do vento.

\section{Resultados}

Seguindo Cuxart et al. (2006), a comparação dos resultados do modelo com os resultados obtidos por Kosovic e Curry (2000), foi feita através da média da nona hora de simulação. É importante lembrar que todas as simulações foram executadas por 10h. Na descrição dos resultados, será adotada uma nomenclatura pra abreviar cada uma das 3 configurações, com 3 diferentes parametrizações utilizadas no modelo. São elas:

- $E-l$ sem função de estabilidade: $E-l$ (Sem FE);

- $E-l$ com função de estabilidade cauda-longa: $E-$ $l$ (FECL);

- $E-l$ com função de estabilidade cauda-curta: $E-$ $l$ (FECC);

- $E-l$ com dissipação modificada sem função de estabilidade: $E-l-\operatorname{Mod}(\mathrm{Sem} F E)$;

- $E-l$ com dissipação modificada com função de estabilidade cauda-longa: $E-l-\operatorname{Mod}(\mathrm{FECL})$;

- $E-l$ com dissipação modificada com função de estabilidade cauda-curta: $E-l-\operatorname{Mod}$ (FECC);

- $E-\varepsilon-l$ sem função de estabilidade: $E-\varepsilon-l$ (Sem FE);

- $E-\varepsilon-l$ com função de estabilidade cauda-longa: $E-\varepsilon-l$ (FECL);

- $E-\varepsilon-l$ com função de estabilidade cauda-curta: $E-\varepsilon-l$ (FECC);

Como já era esperado, a configuração $E-l-M o d$ subestima consideravelmente a intensidade de $\bar{e}$ em toda a CLE, todavia, a altura a qual a turbulência pode chegar é comparável às demais configurações quando fazem uso de FE's (Figura 1a). É importante destacar o papel da utilização da grade com espaçamento variável no modelo, pois enquanto o caso de referência apresenta um máximo de ECT bem localizado (devido ao espaçamento mais grosseiro próximo à superfície), os resultados do modelo apresentam um perfil mais suave. Entretanto, nenhuma das configurações foi capaz de representar adequadamente o valor superfícial de ECT.

Quando o fluxo de calor sensível é observado, Figura $1 \mathrm{~b}$, nota-se que a intensidade deste quando não se faz uso de nenhuma FE na configuração $E-l$, o fluxo de calor sensível chega a quase uma ordem de grandeza superior ao caso do experimento GABLS I. Apesar de menor, o fluxo de calor obtido pela $E-\varepsilon-l$ sem FE é muito superior aos demais casos. A inclusão dos resultados destas duas formulações dificulta a observação do comportamento das demais, logo, neste sentido, a Figura 1c apresenta um ajuste de escala para facilitar tal observação. Na Fig 1c, nota-se o mesmo padrão das demais análises, onde a configuração $E-l-M o d$ sem FE apresenta valores similares das demais configurações com uso de funções de estabilidade, quando uma função 
de estabilidade é usada na configuração $E-l-M o d$, os fluxos turbulentos são reduzidos consideravelmente.

O papel da modificação na parametrização da dissipação viscosa no modelo $E-l$, é observado claramente na Figura 1d. Próximo à superfície, onde o cisalhamento do vento é mais intenso, devido à curvatura do perfil de velocidade, o fluxo de momentum é reduzido consideravelmente, enquanto que, à medida que se observam níveis mais altos, o fluxo de momentum assume valores muito próximos ao da configuração $E-l$. Tal resultado, está de acordo com a proposta de Hunt et al. (1987) para a descrição de um escoamento dominado pelo cisalhamento que diz que, próximo à superfície, o cisalhamento reduz a intensidade turbulenta devido à intensificação da dissipação viscosa.

\section{Conclusões}

As comparações entre os resultados das simulações de Kosovic e Curry (2000) com o modelo aqui apresentado mostram que, de forma geral, todas as configurações e suas respectivas parametrizações, reproduziram a formação de um jato de baixo nível no topo da camada limite. Todavia, entre as configurações e, principalmente, entre as formulações, as maiores diferenças ocorreram na reprodução da altura do jato. Enquanto nas formulações $E-l$ e $E-\varepsilon-l$, ambas sem função de estabilidade (sem FE), a altura do jato foi superestimada em, aproximadamente, $100 \mathrm{~m}$, o uso de uma função de estabilidade cauda curta configuração $E-l-M o d$ (FECC), ocasionou uma subestimação em quase $100 \mathrm{~m}$ na altura da CLE. Entretanto, a configuração $E-l-M o d$ sem FE reproduziu tanto a forma do jato, quanto o perfil de temperatura e a altura de CLE muito próximo das configurações $E-l$ e $E-\varepsilon-l$ com uma função de estabilidade cauda londa (FECL). Isso se deve ao fato que, quando a dissipação é parametrizada em termos do gradiente do vento médio, à medida que a turbulência é reduzida, o gradiente aumenta consecutivamente, aumentando a dissipação, e esta passa a atuar como uma espécie de função de estabilidade.

O efeito da utilização do cisalhamento na parametrização da dissipação de energia cinética turbulenta (ECT) pode ser claramente observado quando os perfils da própria ECT e os perfils dos fluxos turbulentos de momentum e calor sensível são analisados. De maneira geral, próximo à superfície, o gradiente do vento é mais intenso, logo, a dissipação é aumentada e, consecutivamente, a ECT e os fluxos são reduzidos. Como consequência, a velocidade do vento será mais intensa nos primeiros metros acima da superfície, bem como, estas camadas de ar serão mais quentes devido à ausência de mistura. $\mathrm{O}$ panorama se altera à medida que são analisados níveis mais próximos da CLE, pois com o decréscimo do gradiente, a dissipação torna-se menos intensa e, assim, o comportamento das variáveis para o caso $E-l-M o d$ sem FE torna-se comparável à utilização de uma FECL nas outras duas configurações, conforme discutido anteriormente. Ademais, a utilização de uma função de estabilidade na configuração $E-l-M o d$ acarreta na supressão quase que total da turbulência, levando a resultados nada satisfatórios. Apesar disso, estes casos permitem analisar se, em uma situação extrema de turbulência quase que totalmente suprimida, o modelo é capaz de simular o acoplamento atmosférico.

\section{Agradecimentos}

Os autores agradecem às agências CAPES (Coordenação de Pessoal de Nível Superior) e CNPq (Conselho Nacional de Desenvolvimento Científico e Tecnológico) pelo suporte financeiro. O cluster computacional utilizado nas simulações foi adquirido com recursos do Edital 02/2014 - PqG (Edital Pesquisador Gaúcho) da Fundação de Amparo a Pesquisa do Estado do Rio Grande do Sul (FAPERGS).

\section{Referências}

Acevedo, O. C., Costa, F. D., Oliveira, P. E., Puhales, F. S., Degrazia, G. A., Roberti, D. R. (2014). The influence of submeso processes on stable boundary layer similarity relationships. Journal of the Atmospheric Sciences, 71(1), 207-225.

Araújo, A., Nobre, A., Kruijt, B., Elbers, J., Dallarosa, R., Stefani, P., Von Randow, C., Manzi, A., Culf, A., Gash, J., et al. (2002). Comparative measurements of carbon dioxide fluxes from two nearby towers in a central amazonian rainforest: The manaus lba site. Journal of Geophysical Research: Atmospheres (1984-2012), 107(D20), LBA-58.

Belušić, D., Güttler, I. (2010). Can mesoscale models reproduce meandering motions? Quarterly Journal of the Royal Meteorological Society, 136(648), 553-565.

Costa, F. D., Acevedo, O. C., Mombach, J. C. M., Degrazia, G. A. (2011). A simplified model for intermittent turbulence in the nocturnal boundary layer. Journal of the Atmospheric Sciences, 68(8), 1714-1729.

Cuxart, J., Holtslag, A. A., Beare, R. J., Bazile, E., Beljaars, A., Cheng, A., Conangla, L., Ek, M., Freedman, F., Hamdi, R., et al. (2006). Single-column model intercomparison for a stably stratified atmospheric boundary layer. Boundary-Layer Meteorology, 118(2), 273-303. 

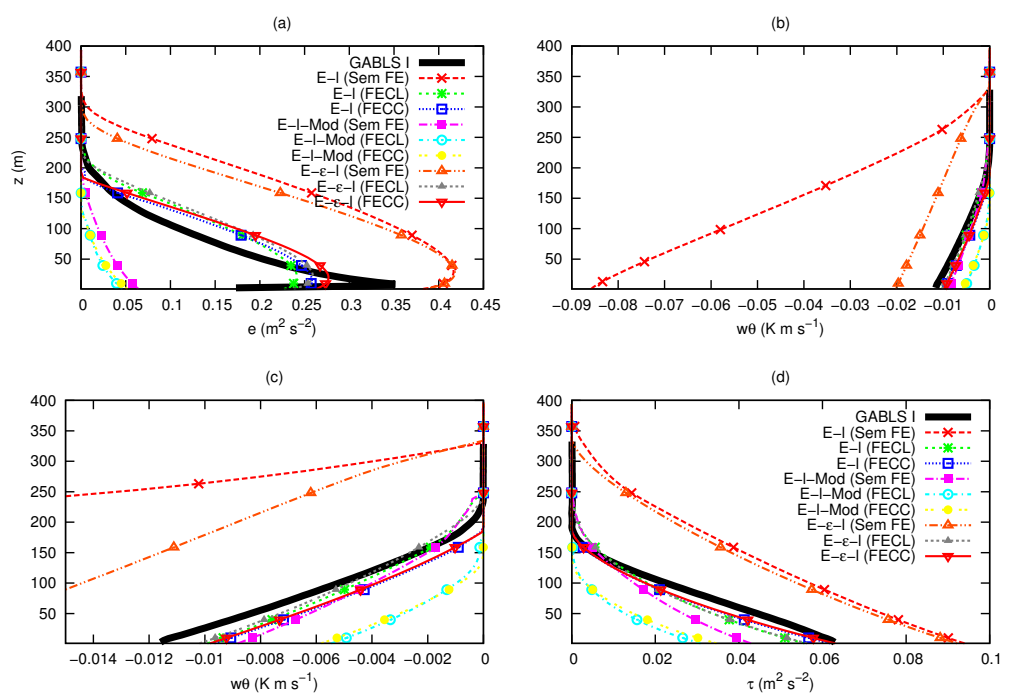

Figura 1: Comparação entre os perfils verticais de ECT(Figura 1a), fluxo de calor sensível (Figura 1b e 1c) e fluxo de momentum por unidade de massa (Figura 1d) para todas as configurações e respectivas formulações, indicadas na legenda, com os resultados de Kosovic e Curry (2000).

Detering, H., Etling, D. (1985). Application of the E- $-\varepsilon$ turbulence model to the atmospheric boundary layer. Boundary-Layer Meteorology, 33(2), 113-133.

Duynkerke, P. (1988). Application of the E- $\varepsilon$ turbulence closure model to the neutral and stable atmospheric boundary layer. Journal of the atmospheric sciences, 45(5), 865-880.

Hunt, J. (1984). Turbulence structure in thermal convection and shear-free boundary layers. Journal of Fluid Mechanics, 138, 161-184.

Hunt, J., Spalart, P., Mansour, N. (1987). A general form for the dissipation length scale in turbulent shear flows. Center for Turbulence Research: Proceedings of the Summer Program 1987.

Kolmogorov, A. N. (1941). Dissipation of energy in locally isotropic turbulence. Doklady Akademii Nauk SSSR, 32(1), 16-18.

Kosovic, B., Curry, J. A. (2000). A large eddy simulation study of a quasi-steady, stably stratified atmospheric boundary layer. Journal of the Atmospheric Sciences, 57(8), 1052-1068.

Mahrt, L. (2009). Characteristics of submeso winds in the stable boundary layer. Boundary-layer meteorology, 130(1), 1-14.

Mahrt, L., Vickers, D. (2005). Boundary-layer adjustment over small-scale changes of surface heat flux. Boundarylayer meteorology, 116(2), 313-330.
Oliveira, P. E., Acevedo, O. C., Moraes, O. L., Zimermann, H. R., Teichrieb, C. (2013). Nocturnal intermittent coupling between the interior of a pine forest and the air above it. Boundary-layer meteorology, 146(1), 45-64.

Puhales, F., Acevedo, O., Degrazia, G., Rizza, U., Moraes, O. (2010). Analysis of the turbulent kinetic energy budget in the planetary boundary layer by large eddy simulation. Em: Extended Abstracts, 19th Symp. on Boundary Layers and Turbulence.

Rodrigo, J. S., Anderson, P. S. (2013). Investigation of the stable atmospheric boundary layer at halley antarctica. Boundary-layer meteorology, 148(3), 517-539.

Sakai, R. K., Fitzjarrald, D. R., Moraes, O. L., Staebler, R. M., Acevedo, O. C., Czikowsky, M. J., Silva, R. d., Brait, E., Miranda, V. (2004). Land-use change effects on local energy, water, and carbon balances in an amazonian agricultural field. Global Change Biology, 10(5), 895-907.

Stull, R. B. (1988). An introduction to boundary layer meteorology, vol 13. Springer.

Teichrieb, C. A., Acevedo, O. C., Degrazia, G. A., Moraes, O. L., Roberti, D. R., Zimermann, H. R., Santos, D. M., Alves, R. C. (2013). Characterizing the relative role of low-frequency and turbulent processes in the nocturnal boundary layer through the analysis of two-point correlations of the wind components. Physica A: Statistical Mechanics and its Applications, 392(6), 1510-1521. 
Weng, W., Taylor, P. A. (2003). On modelling the onedimensional atmospheric boundary layer. Boundarylayer meteorology, 107(2), 371-400.

Weng, W., Taylor, P. A. (2006). Modelling the onedimensional stable boundary layer with an E-1 turbulence closure scheme. Boundary-layer meteorology, $118(2), 305-323$.

Xu, D., Taylor, P. A. (1997). An E-e-1 turbulence closure scheme for planetary boundary-layer models: The neutrally stratified case. Boundary-Layer Meteorology, 84(2), 247-266.

Yuji, K., Akihiro, H., Toshimasa, Y. (2013). Flux richardson number and turbulent prandtl number in a developing stable boundary layer. Journal of the Meteorological Society of Japan, 91(5), 655-666. 